\title{
Vulnerabilidade da pessoa idosa no acesso aos serviços prestados na Atenção Primária
}

Keylla Talitha Fernandes Barbosa ${ }^{1}$, Fabiana Maria Rodrigues Lopes de Oliveira², Maria das Graças Melo Fernandes ${ }^{3}$

\footnotetext{
${ }^{1}$ Enfermeira, Mestre em Enfermagem. Discente do Programa de Pós-Graduação em Enfermagem da Universidade Federal da Paraíba, nível Doutorado. Professora Substituto da Universidade Estadual da Paraíba. João Pessoa, PB, Brasil. E-mail: keyllafernandes@gmail.com.

${ }^{2}$ Enfermeira, Mestre em Enfermagem. Discente do Programa de Pós-Graduação em Enfermagem da Universidade Federal da Paraíba, nível Doutorado. João Pessoa, PB, Brasil. E-mail:

fabianarodriguesenf@yahoo.com.br.

${ }^{3}$ Enfermeira, Doutora em Sociologia. Professora Associado da Universidade Federal da Paraíba. João Pessoa, PB, Brasil. E-mail: graacafernandes@hotmail.com.
}

Recebido: 10/03/2016.

Aceito: 29/03/2017

Publicado: 24/11/2017.

\section{Como citar esse artigo:}

Barbosa KTF, Oliveira FMRL, Fernandes MGM. Vulnerabilidade da pessoa idosa no acesso aos serviços prestados na Atenção Primária. Rev. Eletr. Enf. [Internet]. 2017 [acesso em: _____19:a37. Disponível em: http://dx.doi.org/10.5216/ree.v19.40200.

\section{RESUMO}

Inquérito domiciliar com delineamento descritivo que objetivou identificar a vulnerabilidade programática entre os idosos adstritos à Estratégia Saúde da Família. Estudo realizado com 368 idosos do município de João Pessoa/PB entre os meses de fevereiro e abril de 2014 por meio de entrevista estruturada. Realizou-se a análise descritiva dos dados e o Teste Quiquadrado de Pearson. Dentre a população investigada, 76,6\% não possuía plano privado de saúde e utilizava com maior frequência os serviços disponibilizados pelo Sistema Único de Saúde (96,2\%). Além disso, verificou-se que os idosos residentes em áreas de média vulnerabilidade social apresentaram maior procura por atendimento nas unidades de saúde pública $(96,5 \%)$. Evidenciou-se que a condições sociodemográficas e a vulnerabilidade social podem influenciar diretamente à busca e o acesso aos serviços de saúde pela população idosa.

Descritores: Enfermagem Geriátrica; Vulnerabilidade em Saúde; Atenção Primária à Saúde; Idoso.

\section{INTRODUÇÃO}

O envelhecimento populacional é um fenômeno de amplitude mundial que oportuniza reflexões nos campos social, econômico e epidemiológico, exigindo demandas de políticas públicas que possam proporcionar melhorias na condição de saúde e inserção ativa do idoso na sociedade.

Em virtude do aumento da longevidade e das transformações decorrentes do envelhecimento, como a ocorrência de doenças crônicas não transmissíveis e limitações funcionais, por exemplo, reconhece-se que a procura por serviços de saúde é maior dentre a população idosa(1). Ressalta-se também que, entre os próprios idosos são evidentes as desigualdades no uso de serviços de saúde, de forma que os mais velhos e com menor nível de escolaridade valem-se menos desses serviços, resultando em uma menor atenção preventiva para idosos com piores condições sociais ${ }^{(1)}$. 
Convém destacar que, à medida que aumenta a procura pelos serviços de saúde, há uma evidente necessidade de reorganização do modelo assistencial, buscando-se a ampliação do acesso e a cobertura universal a esses serviços, assim compreendida como a capacidade do sistema de saúde de atender às necessidades da população, incluindo a disponibilidade de infraestrutura, de recursos humanos, de tecnologias da saúde e de financiamento ${ }^{(2)}$. Por meio da ampliação do acesso universal, pode-se eliminar progressivamente as barreiras que impedem a utilização dos serviços de saúde de modo integral por todas as pessoas, particularmente a população idosa, diminuindo as desigualdades de acesso, de prestação de serviços básicos e da vulnerabilidade entre àqueles assistidos ${ }^{(2)}$.

Nessa perspectiva, destaca-se o conceito de vulnerabilidade programática, o qual se refere ao acesso e a forma de organização dos serviços de saúde, incluindo programas voltados para prevenção, assistência e reabilitação ${ }^{(3)}$. Tal dimensão constitui-se na avaliação dos programas para responder ao controle de enfermidades, além do grau e qualidade de compromisso das instituições, dos recursos, da gerência e do monitoramento dos programas nos diferentes níveis de atenção ${ }^{(4)}$. Esse aporte teórico possibilita unir distintos saberes para a construção de uma adequada assistência ao idoso, assim como embasar a elaboração de estratégias de intervenção à saúde de forma integral e resolutiva.

Diante do exposto, delineou-se como objetivo deste estudo identificar a vulnerabilidade programática entre os idosos adstritos à Estratégia Saúde da Família caracterizando-os conforme a utilização dos serviços de saúde.

\section{MÉTODO}

Trata-se de um inquérito domiciliar com delineamento descritivo e corte transversal, desenvolvido entre os idosos assistidos pela Estratégia Saúde da Família do Município de João Pessoa, Paraíba.

A população do estudo compreendeu todos os indivíduos maiores de 60 anos cadastrados no Sistema de Informação da Atenção Básica do referido Município, correspondendo a 24.328 idosos cadastrados em 56 Unidades de Saúde da Família e cinco distritos sanitários. Para a composição da amostra, considerou-se a seguinte fórmula $: n=Z 2 P Q / d 2$, sendo $n=$ tamanho amostral mínimo; $Z$ = variável reduzida; $P=$ probabilidade de encontrar o fenômeno estudado; $Q=1-P ; d$ = precisão desejada, calculado com base em uma margem de erro de $5 \%$ e $p=50 \%$. Utilizou-se a técnica de amostragem estratificada proporcional que levou em consideração os distritos sanitários como estratos.

Foram adotados como critérios de inclusão idosos de ambos os sexos, que evidenciavam condições cognitivas preservadas, de modo que fossem capazes de responder às questões de investigação, bem como aqueles que residiam no distrito sanitário pesquisado. Foram excluídos aqueles que apresentavam déficits de audição e problemas com a fala que dificultassem fortemente a comunicação e impossibilitassem a realização da avaliação. Considerando esses aspectos, a amostra foi constituída por 368 idosos.

A coleta dos dados ocorreu no período de fevereiro a abril de 2014 por discentes com o auxílio logístico dos Agentes Comunitários de Saúde com exercício de atividade laboral nas Unidades de Saúde da Família 
selecionadas. A coleta ocorreu em um único momento nas respectivas residências dos idosos, mediante entrevista estruturada. Para operacionalizar a coleta de informações, foram utilizados três instrumentos: o primeiro, um questionário contemplando variáveis sociodemográficas (idade, sexo, estado civil, anos de estudo, renda familiar). $\mathrm{O}$ segundo compreendeu os indicadores relacionados ao conceito vulnerabilidade programática. 0 terceiro componente avaliado foi o Índice de Vulnerabilidade Social ${ }^{(5)}$, o qual permitiu a identificação do conjunto de famílias e dos territórios em maior vulnerabilidade social, assim como dos componentes do índice que mais contribuíram para esse processo.

Atribuiu-se, a cada componente, um peso a partir da estimativa de risco ou proteção sociossanitária, sendo positivo para os fatores de vulnerabilidade ou negativo para os fatores de proteção. Dessa forma, a vulnerabilidade social é determinada pela ausência ou deficiência das condições positivas e/ou presença de condições negativas para o desenvolvimento das famílias, culminando nas seguintes classificações: sem vulnerabilidade, baixa, média, alta e altíssima vulnerabilidade socia ${ }^{(5)}$.

A análise dos dados foi efetivada numa abordagem quantitativa, por meio da estatística descritiva de natureza univariada para todas as variáveis, incluindo medidas de frequência, de posição e de dispersão. Para comparar as principais variáveis categóricas, foi utilizado o Teste do Qui-quadrado de Pearson com nível de significância estabelecido de 95\%. Para essa análise utilizou-se o sistema computacional Statistical Package for the Social Sciences - SPSS versão 20.0, por ser adequado ao alcance dos objetivos do estudo e por possibilitar a precisão e a generalização dos seus resultados.

Cabe destacar que durante todo o processo da pesquisa foram observados os aspectos éticos que normatizam a pesquisa que envolve seres humanos. Salienta-se também que o projeto de pesquisa em questão foi aprovado pelo Comitê de Ética em Pesquisa do Centro de Ciências da Saúde da Universidade Federal da Paraíba, sob o no de protocolo 0658/13 e CAAE: 23958013.0.0000.5188 de 10 de dezembro de 2013.

\section{RESULTADOS}

No tocante às características sociodemográficas, dos 368 idosos que participaram do estudo, 253 (68,8\%) eram do sexo feminino. A idade variou entre 60 e 103 anos, com média de 71,4 anos e predomínio de idosos na faixa etária entre 60 a 69 (45,9\%). No que diz respeito ao estado civil, 147 (39,9\%) eram casados, com escolaridade entre quatro e oito anos de estudo $(32,6 \%)$ e renda familiar média entre 1,1 e três salários mínimos (80,3\%).

Em relação às variáveis indicadoras de vulnerabilidade programática, evidencia-se que $76,6 \%$ dos idosos não possuem plano médico particular, e apenas $6,8 \%$ dispõem de plano privado odontológico. Dentre os idosos investigados, 96,6\% utilizam o Sistema Único de Saúde, classificando-o como bom (39,5\%) e regular (33,3\%), conforme demonstrado na Tabela 1.

No que diz respeito à procura pelos serviços de saúde, verifica-se que $54,9 \%$ dos idosos buscaram o atendimento médico por três ou mais vezes nos últimos seis meses, com média de 3,1 consultas. No entanto, 
a demanda por serviços odontológicos apresentou-se bastante reduzida, visto que apenas $27,2 \%$ dos idosos declararam procurar o odontólogo no último ano, desse percentual há uma busca predominante pelos profissionais públicos (59\%). Quanto ao acesso às Unidades de Saúde da Família, evidencia-se que 48,4\% da população idosa compareceu às unidades básicas, em média, três ou mais vezes nos últimos seis meses, e $92,4 \%$ obtiveram suporte da equipe de saúde por meio da visita domiciliar.

Tabela 1: Distribuição dos idosos segundo as variáveis indicadoras de vulnerabilidade programática. João Pessoa, PB, Brasil, 2015.

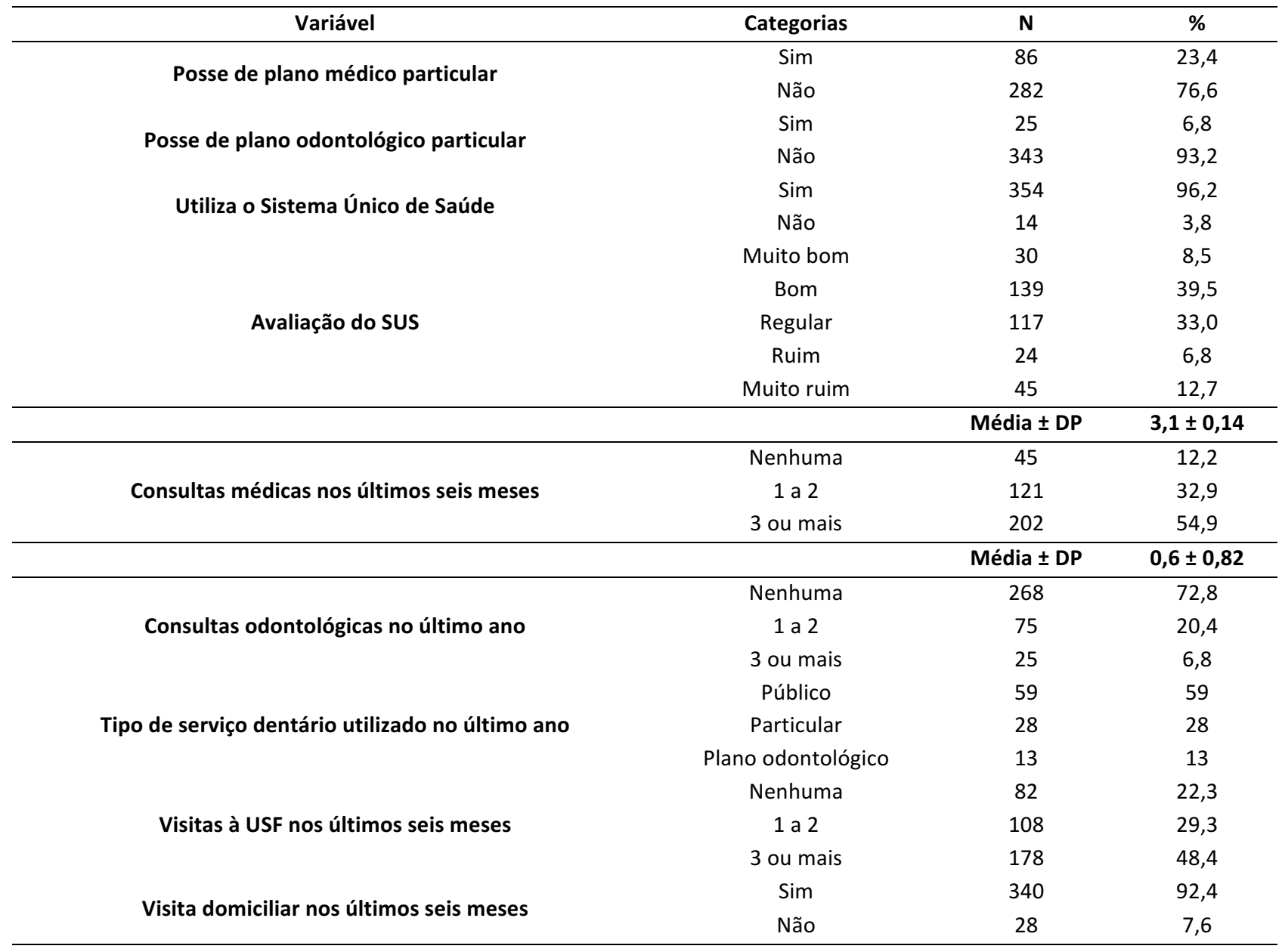

Considerando a associação entre variáveis indicadoras de vulnerabilidade programática e o Índice de Vulnerabilidade Social, percebeu-se que houve associação estatisticamente significativa entre todas as variáveis analisadas, indicando, dessa forma que, em áreas de média vulnerabilidade social, reduziu-se a posse de plano médico $(8,8 \%)$ e de plano odontológico $(1,8 \%)$ privados, refletindo, dessa forma, em maior adesão aos serviços vinculados ao Sistema Único de Saúde (100\%), como, por exemplo, a Unidade de Saúde da Família (96,5\%). Concomitantemente, verificou-se que os idosos residentes em regiões com baixíssimos índices de vulnerabilidade social apresentavam maior adesão aos planos médicos privados $(88,9 \%)$ e odontológicos $(38,2 \%)$. 
Tabela 2: Distribuição dos idosos segundo as variáveis indicadoras de vulnerabilidade programática conforme o IVS. João Pessoa, PB, Brasil, 2015.

\begin{tabular}{|c|c|c|c|c|c|c|c|}
\hline \multirow{3}{*}{ Variável } & \multicolumn{6}{|c|}{ IVS } & \multirow{3}{*}{$\mathbf{p}$} \\
\hline & \multicolumn{2}{|c|}{ Muito baixo } & \multicolumn{2}{|c|}{ Baixo } & \multicolumn{2}{|c|}{ Médio } & \\
\hline & $\mathbf{N}$ & $\%$ & $\mathbf{n}$ & $\%$ & $\mathbf{n}$ & $\%$ & \\
\hline Posse de Plano médico privado & & & & & & & $p<0,001$ \\
\hline Sim & 16 & 88,9 & 65 & 22,2 & 05 & 8,8 & \\
\hline Não & 02 & 11,1 & 228 & 77,8 & 52 & 91,2 & \\
\hline Posse de Plano odontológico privado & & & & & & & $p<0,001$ \\
\hline Sim & 07 & 38,2 & 17 & 5,0 & 01 & 1,8 & \\
\hline Não & 11 & 61,1 & 276 & 94,2 & 56 & 98,2 & \\
\hline Utiliza o SUS & & & & & & & $p<0,001$ \\
\hline Sim & 14 & 77,8 & 283 & 96,6 & 57 & 100 & \\
\hline Não & 04 & 22,2 & 10 & 3,4 & - & - & \\
\hline Frequenta a USF & & & & & & & $p<0,001$ \\
\hline Sim & 12 & 66,7 & 271 & 92,5 & 55 & 96,5 & \\
\hline Não & 06 & 33,3 & 22 & 7,5 & 02 & 3,5 & \\
\hline Total & 18 & 100 & 293 & 100 & 57 & 100 & \\
\hline
\end{tabular}

\section{DISCUSSÃO}

Os indivíduos, especialmente os idosos, enfrentam diferentes situações de vulnerabilidade, individualmente ou coletivamente. Salienta-se que a vulnerabilidade programática trata da influência que as instituições exercem na vida em sociedade e como elas despendem esforços para proteger a população do adoecimento e promover a saúde ${ }^{(6)}$. Dessa forma, avalia a integralidade e a equidade das ações, assim como o acesso aos serviços, a qualidade desses e a existência de equipes multidisciplinares, dialogando harmoniosamente com os princípios do Sistema Único de Saúde ${ }^{(6)}$.

A Constituição Federal Brasileira estabelece a prestação de serviços públicos de modo integral e universal através do Sistema Único de Saúde. Entretanto, diante dos incontáveis obstáculos que constituem a assistência à saúde no Brasil, evidencia-se a crescente busca por cobertura médica suplementar. Apesar de os idosos envolvidos neste estudo possuírem poucos recursos econômicos, constatou-se que $23,4 \%$ deles possuíam plano de assistência médica particular, em consonância com pesquisas semelhantes, o que demonstra possíveis dificuldades vivenciadas por eles com relação ao acesso no atendimento em unidades públicas de saúde de forma resolutiva ${ }^{(1)}$.

A desorganização do sistema público de saúde foi notada como fragilidade, sobretudo, no que diz respeito às dificuldades de acesso, escassez de medicamentos e insumos, assim como a insuficiência de atendimentos especializados. Em decorrência da inabilidade dos serviços prestados pelo Sistema Único de Saúde, a população idosa está aderindo cada vez mais aos planos médicos privados, principalmente devido à segurança quanto ao acesso rápido para atendimento ambulatorial e hospitalar ${ }^{(7)}$. Contudo, acredita-se que as ações disponibilizadas pelas estruturas complementares são predominantemente de caráter curativo e individual. Os planos de saúde foram projetados para pagar despesas médicas agudas e potencialmente devastadoras a curto prazo. Entretanto, à medida que o envelhecimento populacional e as doenças crônicas se tornaram prevalentes, as seguradoras passaram a ofertar, além da assistência médica verticalmente integrada, serviços de saúde, educação e sociais integrados horizontalmente, proporcionando à pessoa idosa 
independência e autonomia por mais tempo possível ${ }^{(8)}$.

Apesar da crescente adesão às estruturas privadas, evidencia-se que o percentual de idosos que utilizam os serviços disponibilizados nas redes conveniadas ao Sistema Único de Saúde é bastante elevado, visto que $96,2 \%$ da população idosa investigada referiram se beneficiar desse atendimento. Ressalta-se que o cuidado ao idoso implica oferecer recursos cuja estrutura apresente características que possibilitem o acesso e o acolhimento adequado a estas pessoas, respeitando suas vulnerabilidades e especificidades, tornando-se um grande desafio para os gestores públicos ${ }^{(9)}$.

Diante da predominância de idosos que utilizam os serviços públicos, deve-se considerar a opinião dos usuários quanto à qualidade da atenção. A participação livre e crítica dos usuários pode contribuir para o empoderamento deste grupo populacional, questões estas essenciais para a viabilização de políticas de promoção da saúde, prevenção de agravos e controle de enfermidades ${ }^{(10)}$. Ao avaliar o Sistema Único de Saúde, verificou-se que 39,5\% dos idosos classificaram-no como bom e 33\%, como regular, dados esses em harmonia com estudo semelhante ${ }^{(10)}$. Tal achado pode ser associado ao fato de que os idosos entrevistados eram usuários da Estratégia Saúde da Família, que geralmente expressam um razoável grau de satisfação em relação ao serviço e a todos os seus atributos: atenção ao primeiro contato, atendimento contínuo e ao longo do tempo, integralidade, coordenação com foco na família e na comunidade ${ }^{(10-11)}$.

A necessidade de manutenção periódica da saúde integral do idoso é evidente, sobretudo a bucal, visto que com o processo de envelhecimento há alterações físicas como retração dos tecidos periodontais, perda do colágeno, tornando-os mais suscetíveis às inflamações no decorrer dos anos, além das alterações mastigatórias decorrentes da perda dos elementos dentários ${ }^{(12)}$. Destaca-se que, $72,8 \%$ dos idosos não havia procurado o odontólogo nenhuma vez no último ano, demonstrando a existência da limitação na utilização dos serviços odontológicos. Visitas regulares ao dentista permitem que os idosos recebam o diagnóstico precoce e cuidados restauradores, conforme necessário. Estudos internacionais recomendam exames orais com periodicidade anual, entretanto evidencia-se que a taxa de utilização do referido serviço tem diminuído de forma constante na última década, elevando os índices de perdas dentárias e demanda crescente por próteses ${ }^{(12-13)}$. A má saúde bucal tem sido associada a condições crônicas de saúde, como diabetes, doenças respiratórias e cardiovasculares. Além disso, demonstram-se que os idosos revelam menor nível de bemestar psicossocial e satisfação com a vida.

Políticas nacionais, estaduais e municipais de saúde, com destaque para atividades de prevenção são fundamentais para a implementação de programas que atendam a saúde do idoso. O sucesso desses programas não depende apenas de ações governamentais, fazendo-se necessária a alteração de valores e atitudes em relação ao cuidado em saúde. Trata-se de apartar da população idosa o estigma de naturalmente doente, ideia que o próprio indivíduo idoso tem de si, para que as necessidades de saúde sejam percebidas e se tornem reais ${ }^{(14)}$.

A criação da Estratégia Saúde da Família como a principal política de estruturação do Sistema Único de Saúde reforça a expansão do acesso aos serviços de saúde, por meio da integralidade das ações individuais 
e coletivas, suscitando um redesenho de modelo assistencial que privilegia o vínculo com a lógica de linhas de cuidado em que todo cidadão tem o direito de ser atendido por uma equipe, de forma integral e resolutiva, com projetos terapêuticos solidários com as demandas dos usuários ${ }^{(8)}$.

No campo da atenção à saúde da pessoa idosa, emerge, dentro das equipes profissionais, a necessidade de criar situações próximas da realidade em que o idoso esteja inserido, de maneira que tais práticas estejam pautadas num olhar crítico e emancipador, conduzindo-o para uma velhice bem sucedida ${ }^{(15)}$. As experiências vivenciadas na Estratégia Saúde da Família permitem caracterizar este estágio da vida como dinâmico, criativo e democrático, por permitir a remodelação constante de práticas e propor o envolvimento do usuário, da família e da comunidade como corresponsáveis nas ações de promoção da saúde ${ }^{(15)}$.

Dentre a população idosa estudada, verificou-se que $48,4 \%$ declararam ter ido à unidade de saúde três vezes ou mais nos últimos seis meses. Os diversos serviços ofertados aos usuários idosos podem influenciar em maior adesão, como consultas com equipe multidisciplinar, atividades de orientações e educação e a possibilidade de participar de grupos de convivência. O gerenciamento abrangente dessa população em um ambiente de atenção primária pode auxiliar a aliviar parte da onerosa carga de prestação de cuidados em um ambiente hospitalar. Torna-se, portanto, um modelo promissor no qual as práticas de saúde são prestadas colaborativamente em um ambiente de cuidados primários ${ }^{(16)}$.

Entretanto, ainda há um predomínio de atividades centradas na doença, contribuindo para fortalecer a cultura de deixar de promover saúde para continuar tratando doentes ${ }^{(17)}$. Um dos instrumentos utilizados que proporciona melhorar as orientações é a visita domiciliar, um modelo para reorganização da assistência, com atividades de controle dos agravos, bem como ações de promoção e de prevenção à saúde. Através dessa ferramenta, a equipe de saúde pode reduzir o número de hospitalizações e o consumo de medicamentos, respeitando o ambiente do cuidado domiciliar, o qual é considerado como o espaço social e físico das interações e interdependências dos seres humanos ${ }^{(17)}$. Ressalta-se que uma das grandes potencialidades da atenção domiciliar é a capacidade da equipe de oferecer suporte e fortalecer as famílias para lidarem com situações críticas, como o envelhecimento, buscando reduzir a sobrecarga e o sofrimento dos usuários ${ }^{(18)}$.

Ainda que a maioria dos idosos investigados no presente estudo tenha recebido visitas domiciliares nos últimos seis meses, verifica-se, em estudos similares, que tais ações mostraram-se numericamente insuficientes, sendo necessário haver visitas mais frequentes e regulares, sobretudo para a população idosa, visto que a complexidade e a multiplicidade dos problemas que eles apresentam demandam maior atenção dos serviços de saúde ${ }^{(17-19)}$. Deve-se estimular o acompanhamento do estado de saúde-doença e cuidados continuados no processo de envelhecimento através de adoção de atitudes interativas, proativas, dialógicas e compartilhadas, buscando recursos para a solução dos problemas de saúde, a melhoria do bem-estar e a qualidade de vida dos idosos no seio da família e da comunidade ${ }^{(20)}$.

Os determinantes sociais da saúde possuem influência no acesso aos serviços em que os idosos residentes em áreas de média vulnerabilidade social apresentavam, no que diz respeito à dimensão 
programática, um perfil distinto daqueles domiciliados em região de muito baixa vulnerabilidade social. Entre a população idosa estudada, observou-se que à medida em que aumentava o Índice de Vulnerabilidade Social a procura por atendimento nas unidades de saúde pública eram mais prevalentes, visto que as condições sociais como o status socioeconômico, o apoio e o engajamento social têm poderosas influências sobre a saúde ${ }^{(21)}$.

Considerando características sociodemográficas, estudos evidenciam que a utilização dos postos ou centros de saúde diminui à medida que aumenta o rendimento familiar mensal, apresentando maior utilização das instituições de saúde privadas entre os idosos de classes econômicas mais privilegiadas e maior grau de escolaridade ${ }^{(19,22)}$. Ressalta-se que, mesmo entre aqueles que possuem planos privados de saúde, há a busca por serviços prestados pelo Sistema Único de Saúde, como vacinas e procedimentos complexos de alto custo, a exemplo da hemodiálise e transplantes ${ }^{(20)}$.

Entretanto, associações entre utilização de serviços de saúde e condições econômicas evidenciam que a probabilidade de os usuários exclusivos do Sistema Único de Saúde residirem em áreas classificadas nos maiores estratos de vulnerabilidade social foi cinco vezes maior se comparada com as demais áreas ${ }^{(22)}$. Apesar de uma maior adesão dos usuários aos serviços públicos, revela-se que o atendimento especializado e exames diagnósticos são precários em decorrência da má integração entre as gestões de nível municipal e estadual, comprometendo o acesso e a qualidade no processo do cuidar ${ }^{(23)}$. A exclusão social em saúde está relacionada também a falta de estrutura e organização dos sistemas de saúde. Em decorrência do abrupto envelhecimento populacional, verifica-se a escassez de programas de suporte e contingência, suscitando a preocupação quanto à capacidade dos sistemas sociais e de saúde em atender a crescente demanda dessa população(22).

Devido aos hábitos acumulados durante a vida, é comum os idosos apresentarem maiores índices de doenças crônicas e incapacidades no decorrer dos anos, resultando em maior procura pelos serviços de saúde e atendimentos hospitalares, contribuindo para a elevação dos gastos por parte dos sistemas de saúde público e privado. Destarte, a população idosa torna-se mais suscetível aos diferentes contextos da vulnerabilidade programática, como a demora por marcações de consulta, encaminhamentos e acompanhamentos para serviços de diferentes densidades tecnológicas, assim como a escassez de atendimento rotineiro com abordagem integral da sua saúde, refletindo em menor acesso à proteção e ao cuidado durante a sua velhice.

Importantes desafios envolvem a reorganização do sistema de saúde brasileiro, sobretudo as esferas relacionadas à Atenção Primária à Saúde. Evidências científicas vêm mostrando que os melhores exemplos de sistemas de saúde são os que possuem uma Atenção Primária à Saúde qualificada e fortalecida, que possa oferecer alcance de maior equidade, maior eficiência na continuidade da atenção e satisfação aos usuários $^{(23)}$. Sob a perspectiva de orientação comunitária no enfretamento de determinantes sociais, redução das desigualdades sociais e regionais, pretende-se fortalecer a Atenção Primária à Saúde e efetivar o direito à saúde no Brasil ${ }^{(24)}$. 
Os enfermeiros constituem-se um instrumento fundamental para o alcance desses objetivos, visto que são profissionais que utilizam uma abordagem integrada e abrangente, estando preparados para responder e gerenciar a saúde ao longo da vida sob a premissa da promoção da saúde, prevenção da doença, tratamento e reabilitação ${ }^{(25)}$. Dentro do contexto da Atenção Primária à Saúde, podem contribuir muito para a redução da morbidade e mortalidade, proporcionando a Atenção Primária à Saúde cuidados iniciais eficazes, visando à manutenção da qualidade de vida em todos os ciclos vitais, sobretudo naqueles mais vulneráveis, como a população idosa ${ }^{(25)}$.

\section{CONCLUSÃO}

O crescente envelhecimento populacional requer adequação dos serviços de saúde por meio do cuidado integral e contextualizado, através do reconhecimento das necessidades individuais e coletivas desse segmento populacional. Para tanto, o presente estudo permitiu identificar que a maioria dos idosos utilizava o Sistema Único de Saúde com uma considerável adesão aos serviços médicos e reduzida procura pelos serviços odontológicos.

Percebeu-se que idosos que residiam em áreas de média vulnerabilidade social possuíam maiores índices de dependência dos serviços públicos de saúde, demonstrando a íntima relação entre os componentes sociodemográficos e o perfil dos serviços utilizados por essa população. Os achados sinalizam a necessidade de planejar as ações de saúde de acordo com os diferentes estratos de vulnerabilidade social, ofertando subsídios para o planejamento de condutas terapêuticas mais próximas da necessidade da população idosa adscrita.

A crescente demanda por serviços públicos que atenda as necessidades reais da população, sobretudo dos idosos, contribui para um dos grandes desafios do Sistema Único de Saúde. A articulação das linhas de cuidado integral por meio da coordenação e organização dos serviços de saúde mostra-se cada vez mais necessária para o provimento da assistência pautada na resolutividade das necessidades dos usuários.

A limitação desse estudo deve-se a sua natureza transversal, em que não é permitido o estabelecimento de relações temporais, bem como os critérios de exclusão adotados que podem ter favorecido a participação dos idosos mais saudáveis e ativos. No entanto, foi possível obter dados que resultaram em informações úteis, as quais poderão ser utilizadas, posteriormente, para subsidiar o desenvolvimento de pesquisas semelhantes na tentativa de elucidar medidas preventivas que promovam o envelhecimento saudável.

Contudo, acredita-se que os resultados obtidos por meio deste estudo representam subsídios relevantes à medida que contribuem para a reflexão por parte dos serviços de saúde, sobretudo, na atenção primária. Os resultados ora demonstrados podem ser utilizados como subsídio para orientação e planejamento de ações que possam reconhecer precocemente o idoso vulnerável, as potencialidades e as fragilidades dos serviços prestadores de cuidados. Futuras investigações sobre o objeto de estudo devem ser encorajadas para se mapear as informações de como a vulnerabilidade na população idosa influencia a 
procura pelos serviços de saúde em diferentes cenários.

\section{REFERÊNCIAS}

1. Pilger C, Menon MU, Mathias TAF. Utilização de serviços de saúde por idosos vivendo na comunidade. Rev Esc Enferm USP. 2013;47(1): 213-20.

2. Organização Pan-Americana da Saúde. Estratégia para o acesso universal à saúde e a cobertura universal de saúde. 2014 [acesso 12 nov 2014]. Disponível em: http://www.paho.org/hq/.

3. Ayres JRCM, França Junior I, Calazans GJ, Saletti Filho HC. O conceito de vulnerabilidade e as práticas de saúde: novas perspectivas e desafios. In: Czeresnia D, Freitas CM. Promoção da saúde: conceitos, reflexões, tendências. Rio de Janeiro: Fiocruz; 2014.

4. Muñoz SAI, Bertolozzi MR. Operacionalização do conceito de vulnerabilidade à tuberculose em alunos universitários. Ciênc Saúde Coletiva. 2011;16(2): 669-75.

5. Fleury-Texeira P. O Índice de Vulnerabilidade Social: IVS Cogitare [CD-ROM]. Belo Horizonte: Biblioteca de Apoio/SG-Al; 2011.

6. Scherer B, Silveira E. Solo e raízes das dimensões individual, social e programática da vulnerabilidade e as sementes no processo de saúde da criança. Texto \& Contexto.2013;12 (1):68-84.

7. Schwartz TD, Ferreira JTB, Maciel ELN, Lima RCD. Estratégia Saúde da Família: avaliando o acesso ao SUS a partir da percepção dos usuários da Unidade de Saúde de Resistência, na região de São Pedro, no município de Vitória (ES). Ciênc Saúde Coletiva. 2010;15(4): 2145-54.

8. Halfon N, Conway PH. The oppoturnities and challenges of a lifelong health. The New England. 2013; 368(17): 156971

9. Oliveira DC, Silva EM. Cuidado ao idoso em Campinas-SP: estudo qualitativo com gestores e representantes políticos. Rev Bras Enferm. 2012; 65(6): 942-9.

10. Moimaz SAS, Marques JAM, Saliba O, Garbin CAS, Zina LG, Saliba NA. Satisfação e percepção do usuário do SUS sobre o serviço público de saúde. Physis. 2010; 20(4):1419-40.

11. Oliveira MAC, Pereira IA. Atributos essenciais da Atenção Primária e a Estratégia Saúde da Família. Rev Bras Enferm. 2013;66 (spe): 158-64.

12. Simões ACA, Carvalho DM. A realidade da saúde bucal do idoso no Sudeste brasileiro. Ciênc Saúde Coletiva. 2011; 16(6): 2975-82.

13. Lee W, Kim SJ, Albert JM, Nelson S. Community factors predicting dental care utilization among older adults. JADA. 2014;145(2):150-8.

14. Rodrigues CAQ, Silva PLV, Caldeira AP, Pordeus IA, Ferreira RC, Martins AMEBL. Fatores associados à satisfação com serviços odontológicos entre idosos. Rev Saúde Pública. 2012;46(6):1039-50.

15. Pinheiro GML, Alvarez AM, Pires DEP. A configuração do trabalho da enfermeira na atenção ao idoso na ESF. Ciênc Saúde Coletiva. 2012;17(8):2105-15.

16. Schiefelbein EL, Olson JÁ, Moxham JD. Patterns of health care utilization among vulnerable populations in central Texas using data from a Regional Health Exchange. J Health Care Poor Undeserved. 2014;25(1):37-51.

17. Gago EA, Lopes MJ. Cuidados domiciliares: interação do enfermeiro com a pessoa idosa/família. Acta Paul Enferm. 2012;25 (spe1):74-80.

18. Burlá C, Camarano AA, Kanso S, Fernandes D, Nunes R. Panorama prospectivo das demências no Brasil: um enfoque demográfico. Ciênc Saúde Coletiva. 2013;18(10): 2949-56.

19. Marin MJS, Bazaglia FC, Massarico AR, Silva CBA, Campos RT, Santos SC. Características sócio-demográficas do atendimento ao idoso após alta hospitalar na Estratégia da Saúde de Família. Rev Esc Enferm USP. 2010; 44(4): 962-8.

20. Polaro SHI, Gonçalves LHT, Alvarez AM. Construindo o fazer gerontológico pelas enfermeiras das Unidades de Estratégia Saúde da Família. Rev Esc Enferm USP. 2013;47(1): 160-7.

21. Andrew MK, Mintnitski A, Kirkland SA, Rockwood K. The impact of social vulnerability on the survival of the fittest older adults. Age and Ageing. 2012;41:161-5.

22. Braga LS, Macinko J, Proietti FA, César CC, Lima-Costa MF. Diferenciais intra-urbanos de vulnerabilidade da população idosa. Cad Saúde Pública. 2010; 26(12): 2307-15. 
23. Paim J, Travassos C, Almeida C, Bahia L, Macinko J. The Brazilian health system:history, advances, and challenges. Lancet. 2011;377(9779):1778-97.

24. Giovanella L. Desafios para Atenção Primária à Saúde. In: Giovanella L, Mendonça MHM, organizadores. Atenção Primária à Saúde: seletiva ou coordenadorados cuidados? Rio de Janeiro: CEBES; 2012. p.74-82

25. Cassiani SHB, Zug KE. Promovendo o papel da Prática Avançada de Enfermagem na América Latina. Rev Bras Enferm. 2014;67(5): 673-6. 